\title{
Ultrahigh-speed non-invasive widefield angiography
}

Cedric Blatter

Thomas Klein

Branislav Grajciar

Tilman Schmoll

Wolfgang Wieser

Raphael Andre

Robert Huber

Rainer A. Leitgeb 


\section{Ultrahigh-speed non-invasive widefield angiography}

\author{
Cedric Blatter, ${ }^{\mathrm{a}}$ Thomas Klein, ${ }^{\mathrm{b}}$ Branislav Grajciar, ${ }^{\mathrm{a}}$ \\ Tilman Schmoll, ${ }^{\mathrm{a}}$ Wolfgang Wieser, ${ }^{\mathrm{b}}$ Raphael Andre, \\ Robert Huber, ${ }^{\prime}$ and Rainer A. Leitgeb ${ }^{a}$ \\ ${ }^{a}$ Medical University Vienna, Center of Medical Physics and Biomedical \\ Engineering, Waehringerguertel 18-20, 1090 Vienna, Austria \\ bLudwig-Maximilians-Universität München, Lehrstuhl für \\ BioMolekulare Optik, Oettingenstraße 67, 80538 Munich, Germany
}

\begin{abstract}
Retinal and choroidal vascular imaging is an important diagnostic benefit for ocular diseases such as age-related macular degeneration. The current gold standard for vessel visualization is fluorescence angiography. We present a potential non-invasive alternative to image blood vessels based on functional Fourier domain optical coherence tomography (OCT). For OCT to compete with the field of view and resolution of angiography while maintaining motion artifacts to a minimum, ultrahigh-speed imaging has to be introduced. We employ Fourier domain mode locking swept source technology that offers high quality imaging at an $A$-scan rate of up to $1.68 \mathrm{MHz}$. We present retinal angiogram over $\sim 48$ deg acquired in a few seconds in a single recording without the need of image stitching. OCT at $1060 \mathrm{~nm}$ allows for high penetration in the choroid and efficient separate characterization of the retinal and choroidal vascularization. $\odot 2012$ Society of Photo-Optical Instrumentation Engineers (SPIE). [DOI: 10.1117/1.JBO.17.7.070505]
\end{abstract}

Keywords: biomedical optics; biophotonics coherent; optical systems; imaging; coherence ophthalmology.

Paper 12222L received Apr. 7, 2012; revised manuscript received Jun. 6, 2012; accepted for publication Jun. 11, 2012; published online Jun. $29,2012$.

Assessment of the retinal and choroidal vascularization yields important diagnostic benefits for major ocular diseases that affect the vascular network already at an early state. The gold standards for their visualization are fluorescein angiography (FA) and indocyanine green angiography. They are commonly used in clinical practice for the diagnosis of vascular occlusions, diabetic retinopathy, and choroidal neovascularization, which is usually a cause of age-related macula degeneration. The invasiveness of these techniques together with undesirable side effects, through the injection of a fluorescent dye, limits the screening capabilities for large populations. Moreover, no depth resolution is available.

Fourier domain optical coherence tomography (FDOCT) is a non-invasive three-dimensional imaging technique with high sensitivity. ${ }^{1}$ Functional extensions of FDOCT and in particular microcirculation imaging have profited from recent technological developments for high-speed FDOCT. ${ }^{2}$ Firstly, images are

Address all correspondence to: Rainer A. Leitgeb, Medical University Vienna Center of Medical Physics and Biomedical Engineering, Group of Biomedical Optics, Waehringerguertel 18-20, 1090 Vienna, Austria. Tel: +43 140400 1714; E-mail: rainer.leitgeb@meduniwien.ac.at virtually free of motion distortion because of smaller acquisition times. Therefore, the patient comfort is improved by keeping measurement duration to a few seconds. Secondly, the lateral sampling can be increased while keeping the optimal $B$-scan rate for vascular contrast imaging. If the $B$-scan rate is low, motion artifacts are more likely to cause unwanted signal decorrelation, reducing the contrast between static tissue and flow. The increase of $B$-scan rate should, however, be limited so that high sensitivity for small capillary flow is preserved. Recent demonstrations applied $B$-scan rates of several hundred $\mathrm{Hz} .{ }^{3}$ Last but not least, a large field of view (FOV) can be covered in a single recording. High-speed FDOCT is thus optimal for non-invasive imaging of retinal microvasculature comprehensively in vivo.

The visualization of the microvasculature yields an easily accessible and intuitive way to assess its integrity. During the last years, a number of strategies for contrasting microvasculature based on FDOCT have been introduced. Contrast between flow and static tissue can be obtained by determining changes either between $A$-scans or successive tomograms in phase, a method known as phase variance, ${ }^{4}$ or in amplitude, known as speckle variance, ${ }^{5}$ or by filtering the complex spectrum, known as OMAG. ${ }^{6}$ Approaches based on phase difference between two spatially separated beams on the retina yielded good contrast of the retinal and choroidal vessel beds over a large range of velocities. ${ }^{7}$ Other authors introduced a pure intensity-based method to filter out capillaries from high resolution data thus avoiding axial decorrelation artifacts below vessels usually present in variance-based contrasting techniques. ${ }^{8}$ Generally, the demonstration of these techniques was restricted to small FOV because of limited acquisition speed. It was partially solved by stitching small volumes together. ${ }^{3}$ A critical point, however, concerning the clinical acceptance of this technique is certainly the associated long total measurement time, because of fixation change and the recording of redundant overlap areas required for registration. Ultrahighspeed FDOCT is therefore a promising candidate to compete with the FOV and resolution of FA, since a large patch can be covered by a single recording in a few seconds. A rough estimation of the required imaging speed, taking into account a spatial sampling of $2000 \times 1000$ pixels, 5-fold redundancy for variation calculation, and a total acquisition time shorter than $10 \mathrm{~s}$, shows that an $A$-scan rate over $1 \mathrm{MHz}$ is required. Recent developments in this imaging speed range are dominated by swept source (SS) systems. Over the last years, multi-MHz imaging was introduced based on the concept of Fourier domain mode locked (FDML) swept sources. Retinal and choroidal imaging with this technology at ultrahigh-speed was demonstrated at a center wavelength of $1060 \mathrm{~nm} .{ }^{9}$ Posterior segment OCT imaging in that water window has the advantage of providing increased penetration into the choroid compared to the common $850 \mathrm{~nm}$ region because of reduced scattering. It allows for a better assessment of choroidal vasculature that is particularly important for ocular diagnosis; its network being the main oxygen and nourishment supplier of the retina.

Angiography using intensity variance analysis is especially suitable for high-speed SS-based systems, because it is independent from any acquisition timing problems, such as trigger jitter.

In the present work we therefore chose to combine ultrahigh-speed FDOCT with intensity variance contrasting for

0091-3286/2012/\$25.00 @ 2012 SPIE 


\section{JBO Letters}

non-invasive depth-resolved angiography of the retina and choroid.

The schematic of the optical setup is the same as previously described for $\mathrm{MHz}$ imaging. ${ }^{9}$ The light source is a FDML laser with its Fabry-Perot filter driven at $\sim 419 \mathrm{kHz}$. The so-called buffering technique of time-multiplexing is later used to increase the sweep rate by a factor of 4 leading to $1.68 \mathrm{MHz} A$-scan rate. The spectrum is centered at $1060 \mathrm{~nm}$ with a $72 \mathrm{~nm}$ sweep range. It produces an $\sim 14 \mu \mathrm{m}$ axial resolution in air. Shot noise limited sensitivity of $\sim 91 \mathrm{~dB}$ with $1.7 \mathrm{~mW}$ power at the cornea is achieved. Widefield imaging is obtained via a sequence of several commercially available, 2 in. diameter planoconvex lenses (Thorlabs), arranged into 2 groups with effective focal lengths of $78 \mathrm{~mm}$ and $41 \mathrm{~mm}$. A $27-\mu \mathrm{m}$ spot size was produced on the retina. The interference signal was measured with a dual-balanced detector (DBD, WieserLabs, WL-BPD1GA) and digitalized with an 8-bit card at 1.5 G Samples/s (Signatec, PX1500-4).

Microcirculation is contrasted against static tissue by calculating first the squared intensity difference between successive tomograms:

$$
P\left(x, y_{i}, z\right)=\left[I\left(x, y_{i}, z\right)-I\left(x, y_{i+1}, z\right)\right]^{2},
$$

where $I(x, y, z)=20 \cdot \log [|\operatorname{FFT}(x, y, k)|]$, and $x, y, z$ are the spatial pixel coordinates corresponding to fast and slow scanning, and the depth coordinate, respectively. The intensity difference tomograms $P$ exhibiting high decorrelation are rejected by comparing their respective mean value to a manually set threshold $T$. The number of pictures averaged at each position can be formally written as

$$
M(y)=\sum_{i=0}^{N-1}\left\{\left[\sum_{x, z} P(x, y, z)\right]<T\right\},
$$

with $N$ as the number of $B$-scans measured at the same position $y$. The value of $T$ is chosen such as to obtain optimal vessel contrast and to suppress residual bulk motion signatures. The logic operation in the brackets yields 1 or 0 for TRUE or FALSE, respectively. Finally, the motion contrast volume $V$ is obtained by averaging only over the remaining $M$ intensity squared difference tomograms $P$ :

$$
V(x, y, z)=\frac{1}{M(y)} \sum_{i=0}^{N-1}\left\{\left[\sum_{x, z} P\left(x, y_{i}, z\right)\right]<T\right\} P\left(x, y_{i}, z\right)
$$

The slow axis scanner is driven by a multiple-step function. It permits measuring successive $B$-scans at the same vertical position $y$, giving an almost perfect correlation for static tissue. We measure $N=5 \mathrm{~B}$-scans at 800 vertical positions. The fast axis driving function is a ramp of $\sim 70 \%$ duty cycle and a frequency of $560 \mathrm{~Hz}$. Each $B$-scan consists of $2060 A$-scans. This leads to an effective volume sampling of $2060 \times 800 \times 460$ ( $x y z)$. The scan amplitude is set to produce an $\sim 48 \mathrm{deg}$ FOV on the retina. The total acquisition time for the full FOV is only $7 \mathrm{~s}$.

Figure 1(a) shows the en-face mean projection of the intensity data set of the retina of a healthy volunteer acquired over a wide FOV of $\sim 48$ deg. Large retinal and choroidal vessels are already visible, however, smaller vessels lack contrast. They are, on the other hand, well appreciated in the high contrast en-face mean projection of the calculated 3-D intensity variance set [Fig. 1(c)]. The FOV of our label-free and non-invasive widefield angiography can be well compared to that of standard
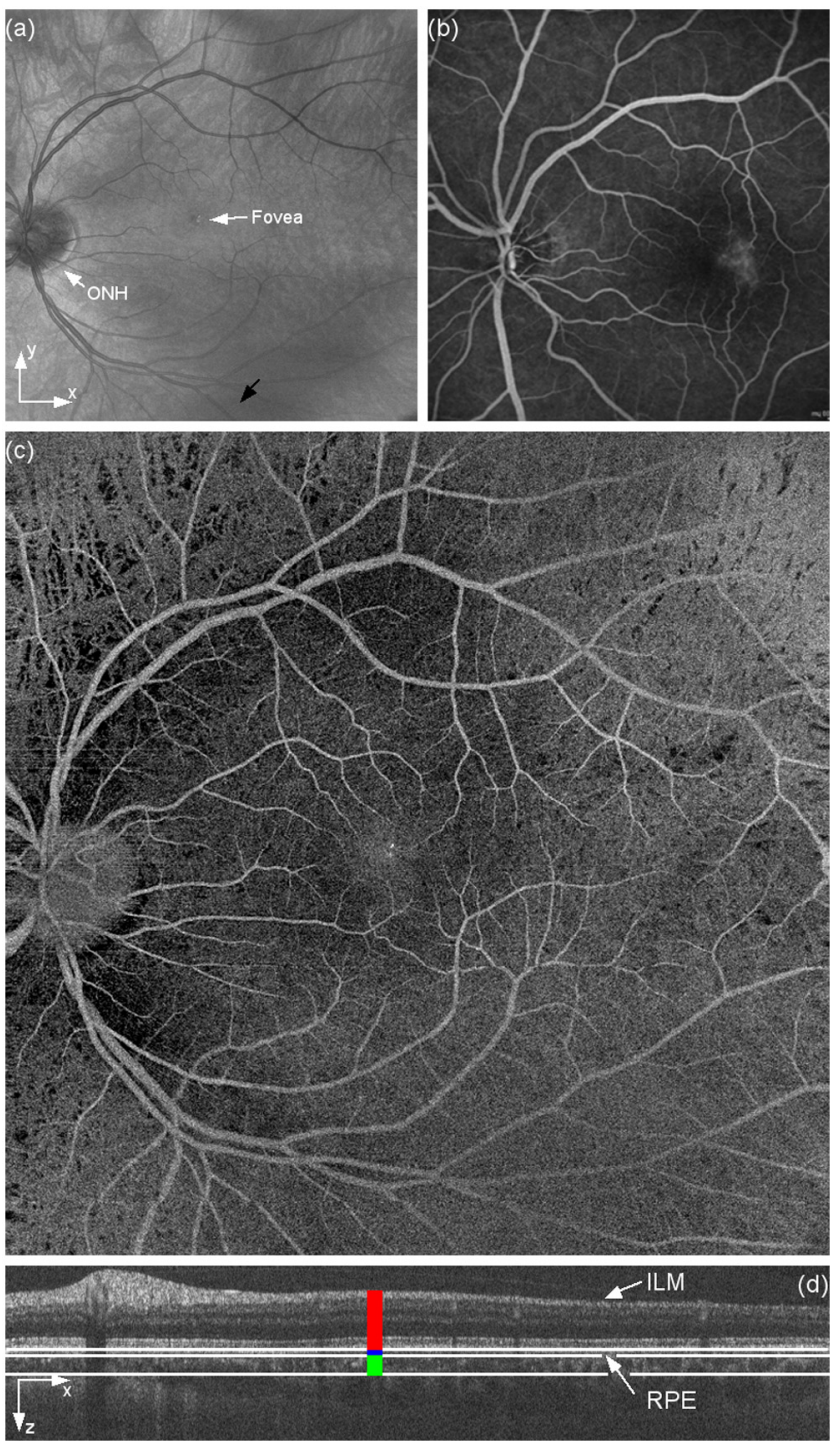

Fig. 1 (a) Pseudo-SLO fundus obtained by en-face mean projection of the intensity data set. ONH: optic nerve head. Black arrow: low signal region. (b) Representative standard fluorescein angiogram of a different subject for comparison (c) $\sim 48$ deg widefield angiogram, en-face mean projection of the intensity difference 3-D data set calculated from (a). (d) Five-fold averaged tomogram after flattening to the retinal pigment epithelium (RPE). Segmentation borders for Fig. 2 are the inner limiting membrane (ILM) and the RPE.

FA. Figure 1(b) shows a standard FA of a patient for qualitative comparison. An important advantage of our technique as compared to previous non-invasive methods based on OCT is the small acquisition time for such image obtained in a single recording.

The depth resolution of OCT allows for differentiating the retinal and choroidal vasculature network for further investigation. For this task, the intensity tomograms were first flattened by detection of the retinal pigment epithelium (RPE) layer [Fig. 1(d)]. In a second step, the position of the inner limiting membrane (ILM) was determined. The corresponding coordinates were used for segmented en-face projection of the intensity difference 3-D set. The first segment consists of the region from ILM to the RPE layer [red bar in Fig. 1(d)], the second segment comprises the vascular structures down to about $50 \mu \mathrm{m}$ below the RPE (blue bar). Large 


\section{JBO Letters}
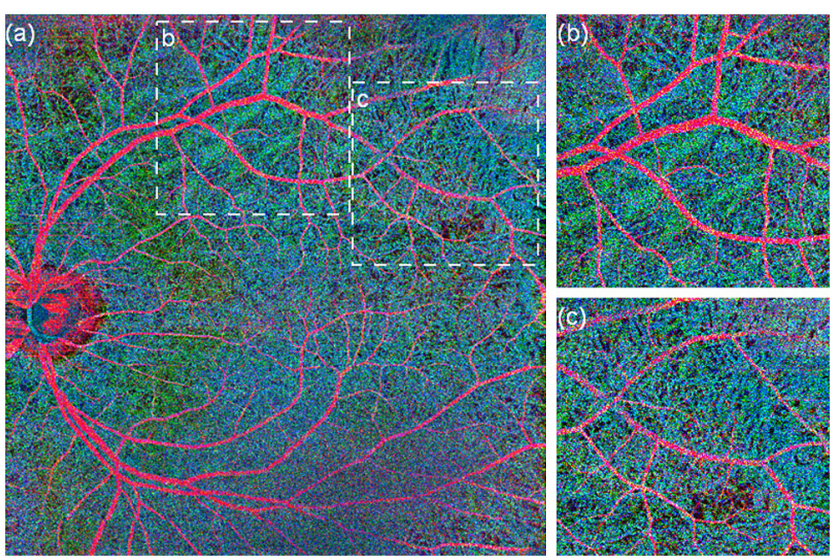

Fig. 2 Color-coded en-face mean projection of the retinal and choroidal vasculature. (a) An 48 deg depth-resolved angiogram. (b) and (c) zoom showing large choroidal vessels and fine vasculature.

choroidal vessels are finally segmented in a third layer (green bar). For the retinal layer, the intensity difference 3-D data set was multiplied by a manually thresholded copy of the intensity data set. It reduces background noise, so that high back-scattered blood vessels are better visible. The segmentation of the $\mathrm{RPE}$ is not possible in the optic nerve head (ONH) region, hence the $\mathrm{ONH}$ vasculature is attributed to the upper layer.

Figure 2 shows a color-coded combined representation of the segmented en-face mean projections for the retina and choroid according to Fig. 1(d). It is possible to resolve, simultaneously, retinal and choroidal vessels. The high lateral sampling allows revealing also small vessels and fine network as shown in Fig. 2(c). The choroid exhibits different kinds of vessels along the depth. Close below the RPE, a dense network of small choroidal vessels is visible [Fig. 2(c)]. In deeper regions, the vessel size increases [Fig. 2(b)]. The low contrast region in the picture's lower part is the result of low signal in the original intensity tomograms [cf. Fig. 1(a), black arrow].

Small retinal capillaries, particularly in the parafoveal region are only weakly visible. This is due to the coarse sampling. Nevertheless, the current protocol would provide a first widefield overview that can then be used as guide to focus on a suspicious area or a region of interest. Figure 3 shows an $\sim 12$ deg FOV centered on the fovea acquired with the same parameters as for the large FOV. The lateral resolution is obviously sufficient to contrast small parafoveal capillaries efficiently [Fig. 3(b) and $3(c)]$ in the en-face mean projection of the intensity difference data set, while absent of the intensity projection [Fig. 3(a)]. Furthermore, choroidal vessels are well appreciated [Fig. 3(d)]. It is, however, difficult to resolve single choroidal vessels in the fovea region due to the dense vascular network directly underneath the RPE. The latter also shades the signatures of deeper choroidal vessels in the en-face view. Faster $B$-scan rates could decrease the difference signal of the choriocapillary layer and enhance the contrast of larger vessels underneath. This has recently been demonstrated employing a dual-beam technique. ${ }^{7}$

In conclusion, single recording with short acquisition time is an important step towards large field label-free and non-invasive angiography. Compared to previous work in vascular imaging using volume stitching, the introduction of ultrahigh-speed allows for small acquisition time, as well as short total measurement duration. It is promising, therefore, as a viable alternative to retinal FA. Still, the latter remains advantageous for the
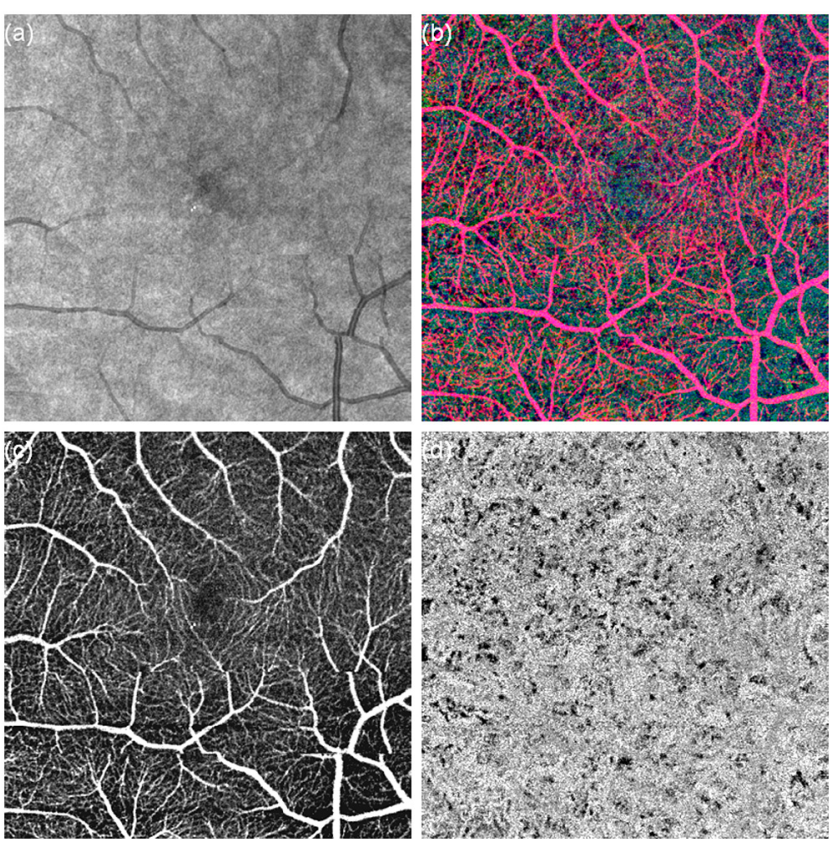

Fig. 3 An $\sim 12$ deg FOV centered at the fovea. (a) Pseudo-SLO fundus obtained by en-face mean projection of the intensity data set. (b) Colorcoded en-face mean projection of the retinal and choroidal vasculature. (c) Retinal vasculature. (d) Choroidal vasculature.

detection of blood leakage, as well as for studying the time evolution of vessel filling.

\section{Acknowledgments}

Funding from the EU FP7 program is acknowledged (FUN OCT, Grant No. 201880). BG is funded by the Austrian Christian Doppler Association.

\section{References}

1. R. Leitgeb, C. Hitzenberger, and A. Fercher, "Performance of Fourier domain vs. time domain optical coherence tomography," Opt. Express 11(8), 889-894 (2003).

2. T. Schmoll, C. Kolbitsch, and R. Leitgeb, "Ultra-high-speed volumetric tomography of human retinal blood flow," Opt. Express 17(5), 4166-4176 (2009).

3. D. Kim et al., "In vivo volumetric imaging of human retinal circulation with phase-variance optical coherence tomography," Biomed. Opt. Express 2(6), 1504-1513 (2011).

4. J. Fingler et al., "Volumetric microvascular imaging of human retina using optical coherence tomography with a novel motion contrast technique," Opt. Express 17(24), 22190-22200 (2009).

5. A. Mariampillai et al., "Speckle variance detection of microvasculature using swept-source optical coherence tomography," Opt. Lett. 33(13), 1530-1532 (2008).

6. R. Wang et al., "Depth-resolved imaging of capillary networks in retina and choroid using ultrahigh sensitive optical microangiography," Opt. Lett. 35(9), 1467-1469 (2010).

7. F. Jaillon, S. Makita, and Y. Yasuno, "Variable velocity range imaging of the choroid with dual-beam optical coherence angiography," Opt. Express 20(1), 385-396 (2012).

8. T. Schmoll et al., "Imaging of the parafoveal capillary network and its integrity analysis using fractal dimension," Biomed. Opt. Express 2(5), 1159-1168 (2011).

9. T. Klein et al., "Megahertz OCT for ultrawide-field retinal imaging with a $1050 \mathrm{~nm}$ Fourier domain mode-locked laser," Opt. Express 19(4), 3044-3062 (2011). 